\title{
Weak Solutions for a $p$-Laplacian Antiperiodic Boundary Value Problem with Impulsive Effects
}

\author{
Keyu Zhang, ${ }^{1,2}{\text { Jiafa } \mathrm{Xu},{ }^{2} \text { and Wei Dong }}^{3}$ \\ ${ }^{1}$ Department of Mathematics, Qilu Normal University, Jinan, Shandong 250013, China \\ ${ }^{2}$ School of Mathematics, Shandong University, Jinan, Shandong 250100, China \\ ${ }^{3}$ Department of Mathematics, Hebei University of Engineering, Handan, Hebei 056038, China
}

Correspondence should be addressed to Jiafa Xu; xujiafa292@sina.com

Received 7 March 2013; Accepted 19 May 2013

Academic Editor: Beatrice Paternoster

Copyright (C) 2013 Keyu Zhang et al. This is an open access article distributed under the Creative Commons Attribution License, which permits unrestricted use, distribution, and reproduction in any medium, provided the original work is properly cited.

By virtue of variational method and critical point theory, we will investigate the existence of weak solutions for a $p$-Laplacian impulsive differential equation with antiperiodic boundary conditions.

\section{Introduction}

In this work, we will study the existence of weak solutions for the $p$-Laplacian impulsive differential equation with antiperiodic boundary conditions

$$
\begin{gathered}
-\left(\left|u^{\prime}\right|^{p-2} u^{\prime}\right)^{\prime}=f(x, u), \quad \text { in } \Omega, \\
\Delta\left|u^{\prime}\left(x_{k}\right)\right|^{p-2} u^{\prime}\left(x_{k}\right)=I_{k}\left(u\left(x_{k}\right)\right), \quad k=1,2, \ldots, m, \\
u(0)=-u(T), \quad u^{\prime}(0)=-u^{\prime}(T),
\end{gathered}
$$

where $p>1, T>0,0=x_{0}<x_{1}<x_{2}<\cdots<x_{m}<$ $x_{m+1}=T, \Omega:=[0, T] \backslash\left\{x_{1}, \ldots, x_{m}\right\}, f \in C([0, T] \times$ $\mathbb{R}, \mathbb{R})$, and $I_{k} \in C(\mathbb{R}, \mathbb{R}), k=1,2, \ldots, m$. Moreover, $\Delta\left|u^{\prime}\left(x_{k}\right)\right|^{p-2} u^{\prime}\left(x_{k}\right)=\left|u^{\prime}\left(x_{k}^{+}\right)\right|^{p-2} u^{\prime}\left(x_{k}^{+}\right)-\left|u^{\prime}\left(x_{k}^{-}\right)\right|^{p-2} u^{\prime}\left(x_{k}^{-}\right)$, where $u^{\prime}\left(x_{k}^{+}\right)$and $u^{\prime}\left(x_{k}^{-}\right)$denote the right and left limits, respectively, of $u^{\prime}(x)$ at $x=x_{k}$, for $k=1,2, \ldots, m$.

Impulsive differential equations have become more important in recent years in some mathematical models of real processes and phenomena studied in physics, chemical technology, population dynamics, biotechnology, and economics. We mention, for instance, the books [1-3] dealing with impulsive differential equations.

Recently, many researchers pay their attention to impulsive differential equations by variational method and critical point theory, to the best our knowledge; we refer the reader to [4-8] and references cited therein. Meanwhile, some people begin to study $p$-Laplacian differential equations with impulsive effects; for example, see [9-14].

Chen and Tang [9] adopted the least action principle and the saddle point theorem to obtain some existence theorems for a second-order $p$-Laplacian system with or without impulsive effects under weak sublinear growth conditions. In [10], they also considered that a second-order impulsive differential equation with Dirichlet problems has one or infinitely many solutions under more relaxed assumptions on their nonlinearity $f$, which satisfies a kind of new superquadratic and subquadratic conditions.

The problem of finding infinitely many large energy solutions is a very classical problem; there is an extensive literature concerning the existence of infinitely many large energy solutions of a plethora of problems via the symmetric mountain pass theorem and fountain theorem; for instance, see $[15-22]$.

Motivated by the works cited above, in this paper, we shall discuss the problem (1). Firstly, we adopt Browder theorem, introduced in [23], to prove that the problem (1) has only one weak solution. Secondly, we shall utilize Fountain theorem under Cerami condition (C), which is introduced in [24], to prove that the problem (1) has infinitely many weak solutions. The results obtained here improve some existing results in the literature. 


\section{Variational Structure}

We first introduce the Banach space $X:=\left\{u \in W^{1, p}([0, T])\right.$ : $u(0)=-u(T)\}$, endowed with the norm

$$
\|u\|=\left(\int_{0}^{T}\left|u^{\prime}(x)\right|^{p} \mathrm{~d} x\right)^{1 / p}, \quad \text { for } u \in X
$$

Then we see that $(X,\|\cdot\|)$ is a reflexive Banach space. We denote by $\|u\|_{p}$ the norm in $L^{p}([0, T])$ and $\|u\|_{\infty}$ in $C[0, T]$.

In what follows, we shall convert the problem (1) into an integral equation. For all $v \in X$, multiply (1) by $v$ and integrate between 0 and $T$ to obtain

$$
\int_{0}^{T}-\left(\left|u^{\prime}(x)\right|^{p-2} u^{\prime}(x)\right)^{\prime} v(x) \mathrm{d} x=\int_{0}^{T} f(x, u(x)) v(x) \mathrm{d} x .
$$

Noting the impulsive effects, for the left integral of (3), we have

$$
\begin{aligned}
\int_{0}^{T}- & \left(\left|u^{\prime}(x)\right|^{p-2} u^{\prime}(x)\right)^{\prime} v(x) \mathrm{d} x \\
= & \sum_{j=0}^{m} \int_{x_{j}^{+}}^{x_{j+1}^{-}}-\left(\left|u^{\prime}(x)\right|^{p-2} u^{\prime}(x)\right)^{\prime} v(x) \mathrm{d} x \\
= & -\left.\sum_{j=0}^{m}\left|u^{\prime}(x)\right|^{p-2} u^{\prime}(x) v(x)\right|_{x_{j}^{+}} ^{x_{j+1}^{-}} \\
& +\sum_{j=0}^{m} \int_{x_{j}^{+}}^{x_{j+1}^{-}}\left|u^{\prime}(x)\right|^{p-2} u^{\prime}(x) v^{\prime}(x) \mathrm{d} x \\
= & \sum_{j=1}^{m}\left[\left|u^{\prime}\left(x_{j}^{+}\right)\right|^{p-2} u^{\prime}\left(x_{j}^{+}\right)-\left|u^{\prime}\left(x_{j}^{-}\right)\right|^{p-2} u^{\prime}\left(x_{j}^{-}\right)\right] v\left(x_{j}\right) \\
& -\left|u^{\prime}(T)\right|^{p-2} u^{\prime}(T) v(T)+\left|u^{\prime}(0)\right|^{p-2} u^{\prime}(0) v(0) \\
& +\int_{0}^{T}\left|u^{\prime}(x)\right|^{p-2} u^{\prime}(x) v^{\prime}(x) \mathrm{d} x \\
= & \sum_{j=1}^{m} I_{j}\left(u\left(x_{j}\right)\right) v\left(x_{j}\right)+\int_{0}^{T}\left|u^{\prime}(x)\right|^{p-2} u^{\prime}(x) v^{\prime}(x) \mathrm{d} x .
\end{aligned}
$$

Substituting (4) into (3), we easily find that

$$
\begin{gathered}
\int_{0}^{T}\left|u^{\prime}(x)\right|^{p-2} u^{\prime}(x) v^{\prime}(x) \mathrm{d} x+\sum_{j=1}^{m} I_{j}\left(u\left(x_{j}\right)\right) v\left(x_{j}\right) \\
-\int_{0}^{T} f(x, u(x)) v(x) \mathrm{d} x=0
\end{gathered}
$$

Definition 1. If for each $v \in X$ there is a $u \in X$ such that (5) holds, then $u \in X$ is called a weak solution for (1).
From (5), we can obtain the weak solutions for (1) that coincide with critical points of the energy functional

$$
\begin{aligned}
J(u)= & \frac{1}{p} \int_{0}^{T}\left|u^{\prime}(x)\right|^{p} \mathrm{~d} x+\sum_{j=1}^{m} \int_{0}^{u\left(x_{j}\right)} I_{j}(x) \mathrm{d} x \\
& -\int_{0}^{T} F(x, u(x)) \mathrm{d} x,
\end{aligned}
$$

where $F(x, u)=\int_{0}^{u} f(x, t) \mathrm{d} t$. Clearly, $J$ is class of $C^{1}$ and its derivative is

$$
\begin{aligned}
\left(J^{\prime}(u), v\right)= & \int_{0}^{T}\left|u^{\prime}(x)\right|^{p-2} u^{\prime}(x) v^{\prime}(x) \mathrm{d} x \\
& +\sum_{j=1}^{m} I_{j}\left(u\left(x_{j}\right)\right) v\left(x_{j}\right) \\
& -\int_{0}^{T} f(x, u(x)) v(x) \mathrm{d} x, \quad \forall u, v \in X .
\end{aligned}
$$

Lemma 2. Consider $X \hookrightarrow \hookrightarrow \quad C[0, T]$ and $X \hookrightarrow \hookrightarrow$ $L^{p}([0, T])$.

Proof. For $u \in X$, by mean value theorem, $u(x)=u(0)+$ $\int_{0}^{x} u^{\prime}(s) \mathrm{d} s$ and $u(x)=u(T)-\int_{x}^{T} u^{\prime}(s) \mathrm{d} s$. It follows from Hölder inequality that

$$
\begin{aligned}
u(x) & =\frac{1}{2}\left[u(0)+\int_{0}^{x} u^{\prime}(s) \mathrm{d} s+u(T)-\int_{x}^{T} u^{\prime}(s) \mathrm{d} s\right] \\
& \leq \frac{1}{2} \int_{0}^{T}\left|u^{\prime}(s)\right| \mathrm{d} s \\
& \leq \frac{1}{2} T^{(p-1) / p}\|u\| .
\end{aligned}
$$

Consequently, we have

$$
\|u\|_{\infty} \leq \frac{1}{2} T^{(p-1) / p}\|u\|, \quad \text { for } u \in X .
$$

Furthermore, for $u \in X$, we have $t, s \in[0, T]$,

$$
|u(t)-u(s)|=\left|\int_{s}^{t} u^{\prime}(\tau) \mathrm{d} \tau\right| \leq \int_{0}^{T}\left|u^{\prime}(\tau)\right| \mathrm{d} \tau \leq T^{(p-1) / p}\|u\| .
$$

Hence every bounded set in $X$ is equicontinuous in $C[0, T]$. By Arzela-Ascoli theorem, we claim that $X \hookrightarrow \hookrightarrow C[0, T]$. By the same manner, we can prove that $X \hookrightarrow \hookrightarrow L^{p}([0, T])$. Furthermore, we can obtain by (9)

$$
\|u\|_{p} \leq \frac{T}{2}\|u\|, \quad \text { for } u \in X
$$

This completes the proof.

Definition 3 (see [23, page 303]). Let $X$ be a reflexive real Banach space and $X^{*}$ its dual. The operator $\mathscr{L}: X \rightarrow X^{*}$ is said to be demicontinuous if $\mathscr{L}$ maps strongly convergent sequences in $X$ to weakly convergent sequences in $X^{*}$. 
Lemma 4 (Browder theorem, see [23, Theorem 5.3.22]). Let $X$ be a reflexive real Banach space. Moreover, let $\mathscr{L}: X \rightarrow X^{*}$ be an operator satisfying the following conditions:

(i) $\mathscr{L}$ is bounded and demicontinuous;

(ii) $\mathscr{L}$ is coercive, that is, $\lim _{\|x\| \rightarrow \infty}(\mathscr{L}(x), x) /\|x\|=+\infty$;

(iii) $\mathscr{L}$ is monotone on the space $X$; that is, for all $x, y \in X$, one has

$$
(\mathscr{L}(x)-\mathscr{L}(y), x-y) \geq 0 \text {. }
$$

Then the equation $\mathscr{L}(x)=f^{*}$ has at least one solution $x \in X$ for every $f^{*} \in X^{*}$. If, moreover, the inequality (12) is strict for all $x, y \in X, x \neq y$, then the equation $\mathscr{L}(x)=f^{*}$ has precisely one solution $x \in X$ for all $f^{*} \in X^{*}$.

In what follows, we shall introduce Fountain theorem under Cerami condition (C). We first give the definition of Cerami condition (C).

Definition 5 (see [24, Definition 1.1]). Assume that $X$ is a Banach space with norm $\|\cdot\|$; we say that $J \in C^{1}(X, \mathbb{R})$ satisfies Cerami condition (C), if for all $d \in \mathbb{R}$,

(i) any bounded sequence $\left\{u_{n}\right\} \subset X$ satisfying $J\left(u_{n}\right) \rightarrow$ $d, J^{\prime}\left(u_{n}\right) \rightarrow 0$ possesses a convergent subsequence;

(ii) there exist $\delta, \xi, \rho>0$ such that for any $u \in J^{-1}([d-$ $\delta, d+\delta])$ with $\|u\| \geq \xi,\left\|J^{\prime}(u)\right\| \cdot\|u\| \geq \rho$.

As $X$ is a reflexive Banach space, there exist (see [25, Section 17]) $\left\{e_{n}\right\}_{n=1}^{\infty} \subset X$ and $\left\{f_{n}\right\}_{n=1}^{\infty} \subset X^{*}$ such that $f_{n}\left(e_{m}\right)=$ $\delta_{n, m}, X=\overline{\operatorname{span}}\left\{e_{n}: n=1,2, \ldots\right\}$ and $X^{*}=\overline{\operatorname{span}}^{W^{*}}\left\{f_{n}: n=\right.$ $1,2, \ldots\}$. For $j, k \in \mathbb{N}$, denote $X_{j}:=\operatorname{span}\left\{e_{j}\right\}, Y_{k}:=\oplus_{j=1}^{k} X_{j}$, and $Z_{k}:=\overline{\oplus_{j=k}^{\infty} X_{j}}$. Clearly, $X=\overline{\oplus_{j \in \mathbb{N}} X_{j}}$ with $\operatorname{dim} X_{j}<\infty$ for all $j \in \mathbb{N}$. Denote $S_{\rho}:=\{u \in X:\|u\|=\rho\}$.

Lemma 6 (see [24, Proposition 1.2]). Let $X, Y_{k}, Z_{k}$ be defined as above. Assume that $J \in C^{1}(X, \mathbb{R})$ satisfies condition $(C)$, and $J(-u)=J(u)$. For each $k \in \mathbb{N}$, there exist $\rho_{k}>r_{k}>0$ such that

(i) $b_{k}:=\inf _{u \in Z_{k} \cap S_{r_{k}}} J(u) \rightarrow+\infty, k \rightarrow \infty$,

(ii) $a_{k}:=\max _{u \in Y_{k} \cap S_{\rho_{k}}} J(u) \leq 0$.

Then $J$ has a sequence of critical points $u_{n}$, such that $J\left(u_{n}\right) \rightarrow+\infty$ as $n \rightarrow \infty$.

\section{Main Results}

Now, we list our assumptions on $f$ and $I_{j}, j=1,2, \ldots, m$.

(H1) $f(x, u)$ is a decreased function about $u$, uniformly in $x \in[0, T]$, and $I_{j}(u)(j=1,2, \ldots, m)$ are increased functions with $u$.

(H2) There exist $a_{j}, b_{j}>0$ and $\gamma_{j} \in[1, p)$ such that $\left|I_{j}(u)\right| \leq$ $a_{j}+b_{j}|u|^{\gamma_{j}-1}$, for all $u \in \mathbb{R}$ and $j=1,2, \ldots, m$.

(H3) There exist $c_{1}, c_{2}>0$ such that $f(x, u) \leq c_{1}+c_{2}|u|^{p-1}$, for all $u \in \mathbb{R}, x \in[0, T]$.
(H4) There is a positive constant $a>0$ such that $\lim _{|u| \rightarrow \infty}(-p F(x, u)+f(x, u) u) /|u| \geq a$, uniformly in $x \in[0, T]$.

(H5) $p \int_{0}^{u} I_{j}(s) \mathrm{d} s-I_{j}(u) u \geq 0, \int_{0}^{u} I_{j}(s) \mathrm{d} s \geq 0$, for all $u \in \mathbb{R}$, $j=1,2, \ldots, m$.

(H6) $\lim _{|u| \rightarrow \infty} F(x, u) /|u|^{p}=+\infty$, uniformly on $x \in$ $[0, T]$.

(H7) $F(x, u)$ is an even function about $u$ and $I_{j}(u)(j=$ $1,2, \ldots, m)$ are odd functions about $u$, for all $x \in$ $[0, T]$.

Theorem 7. Let $0<c_{2}<(T / 2)^{-p}$ and (H1)-(H3) hold. Then (1) has precisely a weak solution.

Proof. For $u, v \in X$, we define

$$
\begin{gathered}
\left(L_{1}(u), v\right):=\int_{0}^{T}\left|u^{\prime}(x)\right|^{p-2} u^{\prime}(x) v^{\prime}(x) \mathrm{d} x, \\
\left(L_{2}(u), v\right):=\sum_{j=1}^{m} I_{j}\left(u\left(x_{j}\right)\right) v\left(x_{j}\right), \\
\left(L_{3}(u), v\right):=\int_{0}^{T} f(x, u(x)) v(x) \mathrm{d} x .
\end{gathered}
$$

Let

$$
\begin{aligned}
(\mathscr{L}(u), v)= & \left(L_{1}(u), v\right)+\left(L_{2}(u), v\right) \\
& -\left(L_{3}(u), v\right), \quad \forall u, v \in X .
\end{aligned}
$$

Then, to find a weak solution of (1) is equivalent to finding a solution for the operator equation $\mathscr{L}(u)=0$. In what follows, we shall sketch the properties of operators $L_{i}(i=1,2,3)$. By Hölder inequality, we have

$$
\begin{aligned}
& \int_{0}^{T}\left|u^{\prime}(x)\right|^{p-2} u^{\prime}(x) v^{\prime}(x) \mathrm{d} x \\
& \leq\left(\left.\left.\int_{0}^{T}|| u^{\prime}(x)\right|^{p-2} u^{\prime}(x)\right|^{p /(p-1)} \mathrm{d} x\right)^{(p-1) / p} \\
& \times\left(\int_{0}^{T}\left|v^{\prime}(x)\right|^{p} \mathrm{~d} x\right)^{1 / p} \\
&=\left(\int_{0}^{T}\left|u^{\prime}(x)\right|^{p} \mathrm{~d} x\right)^{(p-1) / p}\left(\int_{0}^{T}\left|v^{\prime}(x)\right|^{p} \mathrm{~d} x\right)^{1 / p} \\
&=\|u\|^{p-1}\|v\|<\infty, \quad \forall u, v \in X .
\end{aligned}
$$


Consequently, $L_{1}$ is bounded:

$$
\begin{aligned}
& \left|\left(L_{1}\left(u_{n}\right)-L_{1}(u), v\right)\right| \\
& =\left|\int_{0}^{T}\left[\left|u_{n}^{\prime}(x)\right|^{p-2} u_{n}^{\prime}(x)-\left|u^{\prime}(x)\right|^{p-2} u^{\prime}(x)\right] v^{\prime}(x) \mathrm{d} x\right| \\
& \leq\left|\int_{0}^{T}\right|\left|u_{n}^{\prime}(x)\right|^{p-2} u_{n}^{\prime}(x) \\
& \left.\quad-\left.\left|u^{\prime}(x)\right|^{p-2} u^{\prime}(x)\right|^{p /(p-1)} \mathrm{d} x\right)^{(p-1) / p}\|v\| .
\end{aligned}
$$

The last integral tends to zero as $\left\|u_{n}-u\right\| \rightarrow 0$ due to the continuity of the Nemytskii operator $\varphi(s)=|s|^{p-2} s$. Hence, $L_{1}$ is continuous. We assume that $u_{n} \rightarrow u$ in $X$; by Lemma 2 , we know that there is a subsequence, still denoted by $\left\{u_{n}\right\}$, which strongly converges to $u$ in $C[0, T]$. Therefore, utilizing the continuity of $f$, we have

$$
\begin{aligned}
& \left|\left(L_{3}\left(u_{n}\right)-L_{3}(u), v\right)\right| \\
& \quad=\left|\int_{0}^{T}\left[f\left(x, u_{n}(x)\right)-f(x, u(x))\right] v(x) \mathrm{d} x\right| \longrightarrow 0
\end{aligned}
$$

as $n \longrightarrow \infty$.

So, $L_{3}$ is continuous. In the same methods, we see that $L_{2}$ is also continuous. On the other hand, by (9) and (11), for $u, v \in$ $X$, it follows from $(\mathrm{H} 2)$ and $(\mathrm{H} 3)$ that

$$
\begin{aligned}
& \left|\left(L_{2}(u), v\right)\right| \\
& \quad \leq \sum_{j=1}^{m}\left|I_{j}\left(u\left(x_{j}\right)\right)\right|\left|v\left(x_{j}\right)\right| \\
& \quad \leq \sum_{j=1}^{m}\left(a_{j}+b_{j}\left|u\left(x_{j}\right)\right|^{\gamma_{j}-1}\right)\left|v\left(x_{j}\right)\right| \\
& \quad \leq \sum_{j=1}^{m}\left(a_{j}+b_{j}\|u\|_{\infty}^{\gamma_{j}-1}\right)\|v\|_{\infty} \\
& \quad \leq \frac{m}{2} T^{(p-1) / p}\left(a_{j}+b_{j}\left(\frac{1}{2} T^{(p-1) / p}\right)^{\gamma_{j}-1}\|u\|^{\gamma_{j}-1}\right)\|v\| \\
& \quad<\infty,
\end{aligned}
$$

$$
\begin{aligned}
& \left|\left(L_{3}(u), v\right)\right| \\
& \quad=\left|\int_{0}^{T} f(x, u(x)) v(x) \mathrm{d} x\right| \\
& \quad \leq \int_{0}^{T}|f(x, u(x))||v(x)| \mathrm{d} x \\
& \quad \leq \int_{0}^{T}\left(c_{1}+c_{2}|u(x)|^{p-1}\right)|v(x)| \mathrm{d} x \\
& \quad \leq c_{1} T^{(p-1) / p}\|v\|_{p}+c_{2}\|u\|_{p}^{p-1}\|v\|_{p} \\
& \quad \leq \frac{c_{1}}{2} T^{(2 p-1) / p}\|v\|+c_{2}\left(\frac{T}{2}\right)^{p}\|u\|^{p-1}\|v\| \\
& \quad<\infty .
\end{aligned}
$$

Therefore, $L_{2}$ and $L_{3}$ are bounded. Up to now, we have proved that $\mathscr{L}$ is bounded and continuous, so (i) of Lemma 4 holds. Finally, we shall show the monotonicity and coercivity of $\mathscr{L}$. Indeed, (H1) implies that for $u, v \in X$ and $u \neq v$,

$$
\begin{aligned}
& (\mathscr{L}(u)-\mathscr{L}(v), u-v) \\
& =\int_{0}^{T}\left[\left|u^{\prime}(x)\right|^{p-2} u^{\prime}(x)-\left|v^{\prime}(x)\right|^{p-2} v^{\prime}(x)\right] \\
& \quad \times\left(u^{\prime}(x)-v^{\prime}(x)\right) \mathrm{d} x \\
& \quad+\sum_{j=1}^{m}\left(I_{j}\left(u\left(x_{j}\right)\right)-I_{j}\left(v\left(x_{j}\right)\right)\right)\left(u\left(x_{j}\right)-v\left(x_{j}\right)\right) \\
& \quad-\int_{0}^{T}(f(x, u(x))-f(x, v(x)))(u(x)-v(x)) \mathrm{d} x \\
& \geq \int_{0}^{T}\left[\left|u^{\prime}(x)\right|^{p-2} u^{\prime}(x)-\left|v^{\prime}(x)\right|^{p-2} v^{\prime}(x)\right] \\
& \quad \times\left(u^{\prime}(x)-v^{\prime}(x)\right) \mathrm{d} x \\
& \geq\|u\|^{p}-\|u\|^{p-1}\|v\|-\|v\|^{p-1}\|u\|+\|v\|^{p} \\
& \geq\left(\|u\|^{p-1}-\|v\|^{p-1}\right)(\|u\|-\|v\|) \\
& >0 .
\end{aligned}
$$

(H2) and (H3) enable us to get

$$
\begin{aligned}
& (\mathscr{L}(u), u) \\
& =\|u\|^{p}+\sum_{j=1}^{m} I_{j}\left(u\left(x_{j}\right)\right) u\left(x_{j}\right)-\int_{0}^{T} f(x, u(x)) u(x) \mathrm{d} x \\
& \geq\|u\|^{p}-\sum_{j=1}^{m}\left(a_{j}+b_{j}\left|u\left(x_{j}\right)\right|^{\gamma_{j}-1}\right)\left|u\left(x_{j}\right)\right| \\
& \quad-\int_{0}^{T}\left(c_{1}+c_{2}|u(x)|^{p-1}\right)|u(x)| \mathrm{d} x
\end{aligned}
$$




$$
\begin{aligned}
\geq & {\left[1-c_{2}\left(\frac{T}{2}\right)^{p}\right]\|u\|^{p}-\left[\frac{m a_{j}}{2} T^{(p-1) / p}+\frac{c_{1}}{2} T^{(2 p-1) / p}\right]\|u\| } \\
& -m b_{j}\left(\frac{1}{2} T^{(p-1) / p}\right)^{\gamma_{j}}\|u\|^{\gamma_{j}} .
\end{aligned}
$$

Therefore, by the span of $c_{2} \in\left(0,(T / 2)^{-p}\right)$, we arrive at $\lim _{\|u\| \rightarrow \infty}(\mathscr{L}(u), u) /\|u\|=+\infty$. As a result, (ii) and (iii) of Lemma 4 hold. Hence, Lemma 4 implies that (1) has precisely a weak solution. This completes the proof.

Lemma 8. Let (H3)-(H5) hold. Then J satisfies Cerami condition $(C)$.

Proof. For all $d \in \mathbb{R}$, we assume that $\left\{u_{n}\right\}_{n=1}^{\infty} \subset X$ is bounded and

$$
J\left(u_{n}\right) \longrightarrow d, \quad J^{\prime}\left(u_{n}\right) \longrightarrow 0, \quad n \longrightarrow \infty .
$$

Going, if necessary, to a subsequence, we can assume that $u_{n} \rightarrow u$ weakly in $X$, and then

$$
\begin{aligned}
& \left(J^{\prime}\left(u_{n}\right)-J^{\prime}(u), u_{n}-u\right) \\
& =\int_{0}^{T}\left(\left|u_{n}^{\prime}\right|^{p-2} u_{n}^{\prime}-\left|u^{\prime}\right|^{p-2} u^{\prime}\right)\left(u_{n}^{\prime}-u^{\prime}\right) \mathrm{d} x \\
& \quad+\sum_{j=1}^{m}\left(I_{j}\left(u_{n}\left(x_{j}\right)\right)-I_{j}\left(u\left(x_{j}\right)\right)\right)\left(u_{n}\left(x_{j}\right)-u\left(x_{j}\right)\right) \\
& \quad-\int_{0}^{T}\left(f\left(x, u_{n}\right)-f(x, u)\right)\left(u_{n}-u\right) \mathrm{d} x .
\end{aligned}
$$

By Lemma 2, $X \hookrightarrow \hookrightarrow C[0, T]$ enables us to obtain that

$$
\begin{aligned}
& \sum_{j=1}^{m}\left(I_{j}\left(u_{n}\left(x_{j}\right)\right)-I_{j}\left(u\left(x_{j}\right)\right)\right)\left(u_{n}\left(x_{j}\right)-u\left(x_{j}\right)\right) \longrightarrow 0, \\
& \int_{0}^{T}\left(f\left(x, u_{n}\right)-f(x, u)\right)\left(u_{n}-u\right) \mathrm{d} x \longrightarrow 0 \quad \text { as } n \longrightarrow \infty .
\end{aligned}
$$

It follows from $u_{n} \rightarrow u$ weakly in $X$ and $\left(J^{\prime}\left(u_{n}\right)-J^{\prime}(u), u_{n}-\right.$ $u) \rightarrow 0$ that

$$
\int_{0}^{T}\left(\left|u_{n}^{\prime}\right|^{p-2} u_{n}^{\prime}-\left|u^{\prime}\right|^{p-2} u^{\prime}\right)\left(u_{n}^{\prime}-u^{\prime}\right) \mathrm{d} x \longrightarrow 0 \quad \text { as } n \longrightarrow \infty .
$$

Noting (19), we have

$$
\begin{aligned}
& \left(\left\|u_{n}\right\|^{p-1}-\|u\|^{p-1}\right)\left(\left\|u_{n}\right\|-\|u\|\right) \\
& \quad \leq \int_{0}^{T}\left(\left|u_{n}^{\prime}\right|^{p-2} u_{n}^{\prime}-\left|u^{\prime}\right|^{p-2} u^{\prime}\right)\left(u_{n}^{\prime}-u^{\prime}\right) \mathrm{d} x,
\end{aligned}
$$

and thus $\left\|u_{n}-u\right\| \rightarrow 0$ as $n \rightarrow \infty$. Hence, (i) of Definition 5 is satisfied. Next, we prove (ii) of Definition 5; if not, there exists a sequence $\left\{u_{n}\right\} \subset X$ such that

$$
\begin{gathered}
J\left(u_{n}\right) \longrightarrow d, \quad\left\|J^{\prime}\left(u_{n}\right)\right\| \cdot\left\|u_{n}\right\| \longrightarrow 0, \quad n \longrightarrow \infty, \\
\left\|u_{n}\right\| \longrightarrow \infty, \quad n \longrightarrow \infty .
\end{gathered}
$$

By (26), there exists a constant $\varepsilon_{1}>0$ such that

$$
J\left(u_{n}\right)-\frac{1}{p} J^{\prime}\left(u_{n}\right) u_{n} \leq \varepsilon_{1} .
$$

On the other hand, (H4) implies that there is a $M>0$ such that $-p F(x, u)+f(x, u) u \geq a|u|$, for all $|u|>M$ and $x \in$ $[0, T]$. Furthermore, $-p F(x, u)+f(x, u) u$ is bounded for $|u| \leq$ $M$ and $x \in[0, T]$. Therefore, there exists $c>0$ such that $-F(x, u)+(1 / p) f(x, u) u \geq(a / p)|u|-c$, for all $u \in \mathbb{R}, x \in$ $[0, T]$. This, together with (H5), yields

$$
\begin{aligned}
J\left(u_{n}\right)- & \frac{1}{p} J^{\prime}\left(u_{n}\right) u_{n} \\
= & \sum_{j=1}^{m} \int_{0}^{u_{n}\left(x_{j}\right)} I_{j}(x) \mathrm{d} x-\frac{1}{p} I_{j}\left(u_{n}\left(x_{j}\right)\right) u_{n}\left(x_{j}\right) \\
& +\int_{0}^{T}\left(-F\left(x, u_{n}\right)+\frac{1}{p} f\left(x, u_{n}\right) u_{n}\right) \mathrm{d} x \\
\geq & \int_{0}^{T}\left(-F\left(x, u_{n}\right)+\frac{1}{p} f\left(x, u_{n}\right) u_{n}\right) \mathrm{d} x \\
\geq & \int_{0}^{T}\left(\frac{a}{p}\left|u_{n}\right|-c\right) \mathrm{d} x,
\end{aligned}
$$

which implies $\int_{0}^{T}\left|u_{n}\right| \mathrm{d} x \leq p a^{-1}\left(T c+\varepsilon_{1}\right)$. Therefore, there is a $\varepsilon_{2}>0$ such that $\left\|u_{n}\right\|_{\infty} \leq \varepsilon_{2}$.

It follows from (H3) that there are $c_{3}, c_{4}>0$ such that

$$
F(x, u) \leq c_{3}|u|+c_{4}|u|^{p}, \quad \forall u \in \mathbb{R}, x \in[0, T] .
$$

By this and (H5), we can find

$$
\begin{aligned}
J\left(u_{n}\right)= & \frac{1}{p} \int_{0}^{T}\left|u_{n}^{\prime}\right|^{p} \mathrm{~d} x+\sum_{j=1}^{m} \int_{0}^{u_{n}\left(x_{j}\right)} I_{j}(x) \mathrm{d} x \\
& -\int_{0}^{T} F\left(x, u_{n}\right) \mathrm{d} x \\
\geq & \frac{1}{p}\left\|u_{n}\right\|^{p}-\int_{0}^{T}\left(c_{3}\left|u_{n}\right|+c_{4}\left|u_{n}\right|^{p}\right) \mathrm{d} x \\
\geq & \frac{1}{p}\left\|u_{n}\right\|^{p}-c_{3} T\left\|u_{n}\right\|_{\infty}-c_{4} T\left\|u_{n}\right\|_{\infty}^{p} \\
\geq & \frac{1}{p}\left\|u_{n}\right\|^{p}-c_{3} T \varepsilon_{2}-c_{4} T \varepsilon_{2}^{p},
\end{aligned}
$$


and thus $J\left(u_{n}\right) \rightarrow \infty$ if (27) holds, which contradicts $J\left(u_{n}\right) \rightarrow d$ in (26). This proves that $J$ satisfies condition (C).

Theorem 9. Suppose that (H2)-(H7) hold, and then (1) has infinitely many weak solutions.

Proof. By (H7), we know that $J$ is even. Denote $\beta_{k}=$ $\sup _{u \in Z_{k} \cap S_{1}}\|u\|_{p}$, and by the compactness of the embedding $X \hookrightarrow \hookrightarrow L^{p}([0, T])$, we see that $\beta_{k} \rightarrow 0$ as $k \rightarrow \infty$ (see [26, Lemma 3.8]). Noting (30), we have by (H5) and Hölder inequality, for any $u \in Z_{k}$ and $\|u\|=r_{k}:=\beta_{k}^{-1}$,

$$
\begin{aligned}
J(u) & \geq \frac{1}{p}\|u\|^{p}-\int_{0}^{T}\left(c_{3}|u|+c_{4}|u|^{p}\right) \mathrm{d} x \\
& \geq \frac{1}{p}\|u\|^{p}-c_{3} T^{(p-1) / p}\|u\|_{p}-c_{4}\|u\|_{p}^{p} \\
& \geq \frac{1}{p}\|u\|^{p}-c_{3} T^{(p-1) / p} \beta_{k}\|u\|-c_{4} \beta_{k}^{p}\|u\|^{p} \\
& \geq \frac{\beta_{k}^{-p}}{p}-c_{3} T^{(p-1) / p}-c_{4} .
\end{aligned}
$$

We easily have $r_{k} \rightarrow \infty$ as $k \rightarrow \infty$ and $J(u) \geq \beta_{k}^{-p} / p-$ $c_{3} T^{(p-1) / p}-c_{4} \rightarrow \infty$ as $k \rightarrow \infty$. Hence,

$$
b_{k}:=\inf _{u \in Z_{k},\|u\|=r_{k}} J(u) \longrightarrow \infty \quad \text { as } k \longrightarrow \infty
$$

On the other hand, by (H6), we find that there are $b, c>0$ such that

$$
F(x, u) \geq b|u|^{p}-c, \quad \forall u \in \mathbb{R}, x \in[0, T] .
$$

Since all the norms of a finite dimensional normed space are equivalent, note that $\|\cdot\|_{p}$ is a norm of $Y_{k}$, so there exists a $\xi>0$ such that

$$
\|u\|_{p}^{p} \geq \xi\|u\|^{p}, \quad \forall u \in Y_{k} .
$$

Noting (9), we have

$$
\begin{aligned}
J(u)= & \frac{1}{p} \int_{0}^{T}\left|u^{\prime}\right|^{p} \mathrm{~d} x+\sum_{j=1}^{m} \int_{0}^{u\left(x_{j}\right)} I_{j}(x) \mathrm{d} x-\int_{0}^{T} F(x, u) \mathrm{d} x \\
\leq & \frac{1}{p}\|u\|^{p}-\int_{0}^{T}\left(b|u|^{p}-c\right) \mathrm{d} x \\
& +\sum_{j=1}^{m} \int_{0}^{u\left(x_{j}\right)}\left(a_{j}+b_{j}|s|^{\gamma_{j}-1}\right) \mathrm{d} x
\end{aligned}
$$

$$
\begin{aligned}
\leq & \frac{1}{p}\|u\|^{p}-b\|u\|_{p}^{p}+c T \\
& +\sum_{j=1}^{m}\left[a_{j}\left|u\left(x_{j}\right)\right|+\frac{b_{j}}{\gamma_{j}}\left|u\left(x_{j}\right)\right|^{\gamma_{j}}\right] \\
\leq & \frac{1}{p}\|u\|^{p}-b \xi\|u\|^{p}+\sum_{j=1}^{m}\left[a_{j}\|u\|_{\infty}+\frac{b_{j}}{\gamma_{j}}\|u\|_{\infty}^{\gamma_{j}}\right]+c T \\
\leq & \left(\frac{1}{p}-b \xi\right)\|u\|^{p} \\
& +\sum_{j=1}^{m}\left[\frac{a_{j}}{2} T^{(p-1) / p}\|u\|+\frac{b_{j}}{\gamma_{j}}\left(\frac{1}{2} T^{(p-1) / p}\right)^{\gamma_{j}}\|u\|^{\gamma_{j}}\right]+c T .
\end{aligned}
$$

Note that we can choose a large enough $b$ such that $1 / p-b \xi<$ 0 by (H6) and $p>\gamma_{j}$ by (H2), and then there exists positive constants $d_{k}$ such that

$$
J(u) \leq 0, \quad \text { for each } u \in Y_{k},\|u\| \geq d_{k} .
$$

By this and (33), we can take $\rho_{k}:=\max \left\{d_{k}, r_{k}+1\right\}$, and thus $a_{k}:=\max _{u \in Y_{k},\|u\|=\rho_{k}} J(u) \leq 0$. Up until now, we have proved that the functional $J$ satisfies all the conditions of Lemma 6, and then $J$ has infinitely many critical points. Equivalently, (1) has infinitely many weak solutions. This completes the proof.

Two Examples. (1) Let $p=4$ and $x_{1}=T / 2$. Consider the problem

$$
\begin{gathered}
-\left(\left|u^{\prime}\right|^{2} u^{\prime}\right)^{\prime}=f(x, u), \quad \text { in } \Omega, \\
\Delta\left|u^{\prime}\left(x_{1}\right)\right|^{2} u^{\prime}\left(x_{1}\right)=u\left(x_{1}\right), \\
u(0)=-u(T), \quad u^{\prime}(0)=-u^{\prime}(T),
\end{gathered}
$$

where $f(x, u)=-\eta_{1}-\eta_{2} u^{3}, \eta_{1} \geq 0, \eta_{2} \in\left(0,(T / 2)^{-4}\right)$. Clearly, (H1)-(H3) hold true. By Theorem 7, (38) has only a weak solution.

(2) (H6) can be weaken that

$$
\lim _{|u| \rightarrow \infty} \frac{F(x, u)}{|u|^{p}}>(p \xi)^{-1}, \quad \text { uniformly on } x \in[0, T] \text {, }
$$

where $\xi$ is determined by (35). Indeed, by (39), we can obtain that there exist $b>(p \xi)^{-1}$ and $c>0$ such that (34) is satisfied. Furthermore, $1 / p-b \xi<0$.

Let $p=4$ and $x_{1}=T / 2$. Consider the problem

$$
\begin{gathered}
-\left(\left|u^{\prime}\right|^{2} u^{\prime}\right)^{\prime}=f(x, u), \quad \text { in } \Omega, \\
\Delta\left|u^{\prime}\left(x_{1}\right)\right|^{2} u^{\prime}\left(x_{1}\right)=\sqrt[3]{u\left(x_{1}\right)}, \\
u(0)=-u(T), \quad u^{\prime}(0)=-u^{\prime}(T),
\end{gathered}
$$

where $f(x, u)=-\eta+\left(\xi^{-1}+4\right) u^{3}$ and $I_{j}\left(u\left(x_{j}\right)\right)=\sqrt[3]{u\left(x_{1}\right)}$, where $\eta>0$ and $\xi$ is defined by (35). 
For $I_{j}(u)$, we can easily have $(\mathrm{H} 2)$ and $(\mathrm{H} 7)$ hold. By computation, $\int_{0}^{u} I_{j}(s) \mathrm{d} s=(3 / 4) \sqrt[3]{u^{4}} \geq 0, p \int_{0}^{u} I_{j}(s) \mathrm{d} s-$ $I_{j}(u) u=4 \times(3 / 4) \sqrt[3]{u^{4}}-\sqrt[3]{u^{4}}=2 \sqrt[3]{u^{4}} \geq 0$, for all $u \in \mathbb{R}$, and hence we obtain that (H5) is satisfied.

For $f(x, u)$ and $F(x, u)$, we see $f(x, u)=-\eta+\left(\xi^{-1}+4\right) u^{3} \leq$ $\eta+\left(\xi^{-1}+4\right)|u|^{3}$, for all $u \in \mathbb{R}, x \in[0, T]$. Therefore, (H3) holds.

It is obvious that $F(x, u)=\int_{0}^{u} f(x, s) \mathrm{d} s=\int_{0}^{u}\left(-\eta+\left(\xi^{-1}+\right.\right.$ $\left.4) s^{3}\right) \mathrm{d} s=-\eta|u|+\left(\left(\xi^{-1}+4\right) / 4\right)|u|^{4}$, so (H7) holds; furthermore,

$$
\begin{aligned}
\lim _{|u| \rightarrow \infty} \frac{F(x, u)}{|u|^{p}} & =\lim _{|u| \rightarrow \infty} \frac{-\eta|u|+\left(\left(\xi^{-1}+4\right) / 4\right)|u|^{4}}{|u|^{4}} \\
& =\frac{\xi^{-1}+4}{4}>(p \xi)^{-1} .
\end{aligned}
$$

As a result of this, (39) (i.e., (H6)) is true.

By

$$
\begin{aligned}
- & p F(x, u)+f(x, u) u \\
& =-4\left[-\eta|u|+\frac{\xi^{-1}+4}{4}|u|^{4}\right]+\left(-\eta+\left(\xi^{-1}+4\right) u^{3}\right) u \\
& =4 \eta|u|-\eta u \\
& \geq 3 \eta|u|,
\end{aligned}
$$

we obtain that

$$
\lim _{|u| \rightarrow \infty} \frac{-p F(x, u)+f(x, u) u}{|u|} \geq \lim _{|u| \rightarrow \infty} \frac{3 \eta|u|}{|u|}=3 \eta,
$$

uniformly on $x \in[0, T]$. Consequently, (H4) holds. Nowadays, we have proved that (H2)-(H7) hold, and then (40) has infinitely many weak solutions by Theorem 9 .

In [14], the condition $\left(p_{2}\right)$ is

$$
\exists \mu>p \quad \exists \delta>0: \forall|t| \geq \delta, \quad 0 \leq \mu F(x, t) \leq t f(x, t) .
$$

As known to all, this condition is originally introduced in [27] and is still present in many works which is used to guarantee the boundedness of (P.S.) sequences of the corresponding functional.

In the problem (40), note that there exists $\delta>0$ such that $|u| \geq \delta$ and

$$
\begin{aligned}
\mu F(x, u) & \leq u f(x, u) \Longrightarrow-\eta \mu|u|+\frac{\xi^{-1}+4}{4} \mu|u|^{4} \\
& \leq-\eta u+\left(\xi^{-1}+4\right)|u|^{4} .
\end{aligned}
$$

This is impossible if $\mu>p=4$ and $\delta$ is an adequately large positive number. On the other hand, if (H4) holds, then there is a $\delta$ sufficiently large such that

$$
-p F(x, u)+u f(x, u) \geq a|u| \geq 0, \quad \text { for } x \in[0, T],|u| \geq \delta \text {. }
$$

Therefore, $p F(x, u) \leq u f(x, u)$, for all $x \in[0, T]$ and $|u| \geq$ $\delta$, which is also weaker than the condition $\left(p_{2}\right)$ if $F(x, u) \geq$ 0 . This means that our results generalize the corresponding results in [14].

\section{Acknowledgments}

This research is supported by the NNSF China (10971046), Shandong and Hebei Provincial Natural Science Foundation (ZR2012AQ007, A2012402036), GIIFSDU (yzc12063), IIFSDU (2012TS020), and the Project of Shandong Province Higher Educational Science and Technology Program (J09LA55).

\section{References}

[1] A. M. Samoilenko and N. A. Perestyuk, Impulsive Differential Equations, vol. 14 of World Scientific Series on Nonlinear Science. Series A: Monographs and Treatises, World Scientific, River Edge, NJ, USA, 1995.

[2] V. Lakshmikantham, D. D. Bainov, and P. S. Simeonov, Theory of Impulsive Differential Equations, vol. 6 of Series in Modern Applied Mathematics, World Scientific, Teaneck, NJ, USA, 1989.

[3] M. Benchohra, J. Henderson, and S. Ntouyas, Impulsive Differential Equations and Inclusions, vol. 2 of Contemporary Mathematics and Its Applications, Hindawi Publishing Corporation, New York, NY, USA, 2006.

[4] J. J. Nieto and D. O'Regan, "Variational approach to impulsive differential equations," Nonlinear Analysis. Real World Applications, vol. 10, no. 2, pp. 680-690, 2009.

[5] J. Sun, H. Chen, J. J. Nieto, and M. Otero-Novoa, "The multiplicity of solutions for perturbed second-order Hamiltonian systems with impulsive effects," Nonlinear Analysis. Theory, Methods \& Applications, vol. 72, no. 12, pp. 4575-4586, 2010.

[6] Y. Tian, W. Ge, and D. Yang, "Existence results for second-order system with impulse effects via variational methods," Journal of Applied Mathematics and Computing, vol. 31, no. 1-2, pp. 255$265,2009$.

[7] J. Xiao, J. J. Nieto, and Z. Luo, "Multiplicity of solutions for nonlinear second order impulsive differential equations with linear derivative dependence via variational methods," Communications in Nonlinear Science and Numerical Simulation, vol. 17, no. 1, pp. 426-432, 2012.

[8] J. Zhou and Y. Li, "Existence of solutions for a class of secondorder Hamiltonian systems with impulsive effects," Nonlinear Analysis. Theory, Methods \& Applications, vol. 72, no. 3-4, pp. 1594-1603, 2010.

[9] P. Chen and X. H. Tang, "Existence of solutions for a class of $p$ Laplacian systems with impulsive effects," Taiwanese Journal of Mathematics, vol. 16, no. 3, pp. 803-828, 2012.

[10] P. Chen and X. Tang, "Existence and multiplicity of solutions for second-order impulsive differential equations with Dirichlet problems," Applied Mathematics and Computation, vol. 218, no. 24, pp. 11775-11789, 2012.

[11] L. Bai and B. Dai, "Three solutions for a $p$-Laplacian boundary value problem with impulsive effects," Applied Mathematics and Computation, vol. 217, no. 24, pp. 9895-9904, 2011.

[12] L. Bai and B. Dai, "Existence and multiplicity of solutions for an impulsive boundary value problem with a parameter via critical point theory," Mathematical and Computer Modelling, vol. 53, no. 9-10, pp. 1844-1855, 2011.

[13] L. Wang, W. Ge, and M. Pei, "Infinitely many solutions of a second-order $p$-Laplacian problem with impulsive condition," Applications of Mathematics, vol. 55, no. 5, pp. 405-418, 2010.

[14] I. Bogun, "Existence of weak solutions for impulsive $p$ Laplacian problem with superlinear impulses," Nonlinear Analysis. Real World Applications, vol. 13, no. 6, pp. 2701-2707, 2012. 
[15] T. Bartsch, "Infinitely many solutions of a symmetric Dirichlet problem," Nonlinear Analysis. Theory, Methods \& Applications, vol. 20, no. 10, pp. 1205-1216, 1993.

[16] T. Bartsch and M. Willem, "On an elliptic equation with concave and convex nonlinearities," Proceedings of the American Mathematical Society, vol. 123, no. 11, pp. 3555-3561, 1995.

[17] J. Chen and X. Tang, "Infinitely many solutions for a class of fractional boundary value problem," http://www.emis.de/ journals/BMMSS/pdf/acceptedpapers/2011-09-043_R1.pdf.

[18] L. Zhang, X. H. Tang, and J. Chen, "Infinitely many periodic solutions for some second-order differential systems with $p(t)$ Laplacian," Boundary Value Problems, vol. 33, 15 pages, 2011.

[19] U. B. Severo, "Multiplicity of solutions for a class of quasilinear elliptic equations with concave and convex terms in $\mathbb{R}$, , Electronic Journal of Qualitative Theory of Differential Equations, vol. 5, pp. 1-16, 2008.

[20] S. Yan and J. Yang, "Fountain theorem over cones and applications," Acta Mathematica Scientia B, vol. 30, no. 6, pp. 1881-1888, 2010.

[21] D. Liu, "On a $p$-Kirchhoff equation via fountain theorem and dual fountain theorem," Nonlinear Analysis. Theory, Methods \& Applications, vol. 72, no. 1, pp. 302-308, 2010.

[22] P. S. Iliaş, "Existence and multiplicity of solutions of a $p(x)$ Laplacian equation in a bounded domain," Revue Roumaine de Mathématiques Pures et Appliquées, vol. 52, no. 6, pp. 639-653, 2007.

[23] P. Drábek and J. Milota, Methods of Nonlinear Analysis: Applications to Differential Equations, Birkhäuser Advanced Texts: Basler Lehrbücher, Birkhäuser, Basel, Switzerland, 2007.

[24] A. Qian and C. Li, "Infinitely many solutions for a Robin boundary value problem," International Journal of Differential Equations, vol. 2010, Article ID 548702, 9 pages, 2010.

[25] J. Zhao, Structure Theory of Banach Spaces, Wuhan University Press, Wuhan, China, 1991.

[26] M. Willem, Minimax Theorems, Progress in Nonlinear Differential Equations and their Applications, Birkhäuser, Boston, Mass, USA, 1996.

[27] A. Ambrosetti and P. H. Rabinowitz, "Dual variational methods in critical point theory and applications," Journal of Functional Analysis, vol. 14, pp. 349-381, 1973. 


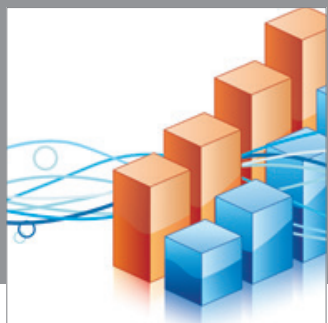

Advances in

Operations Research

mansans

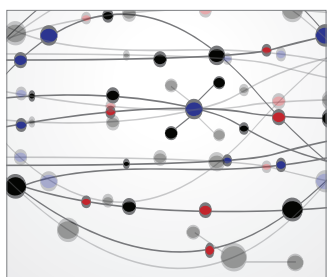

The Scientific World Journal
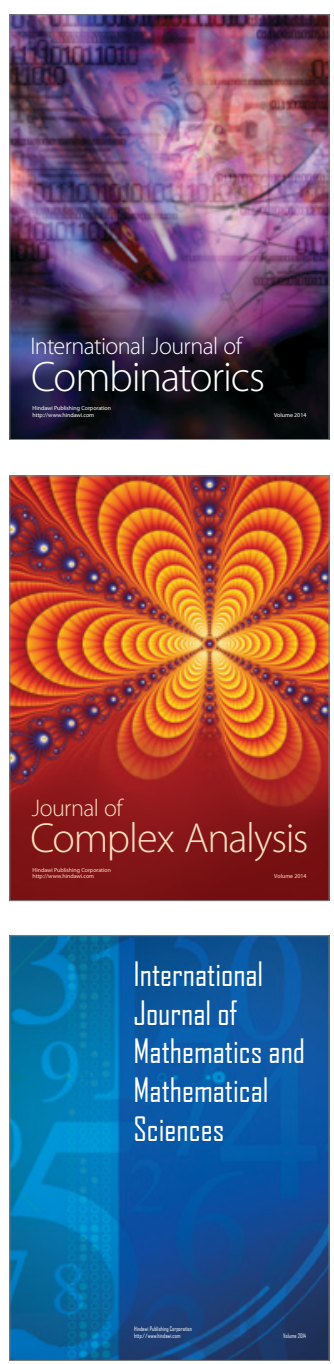
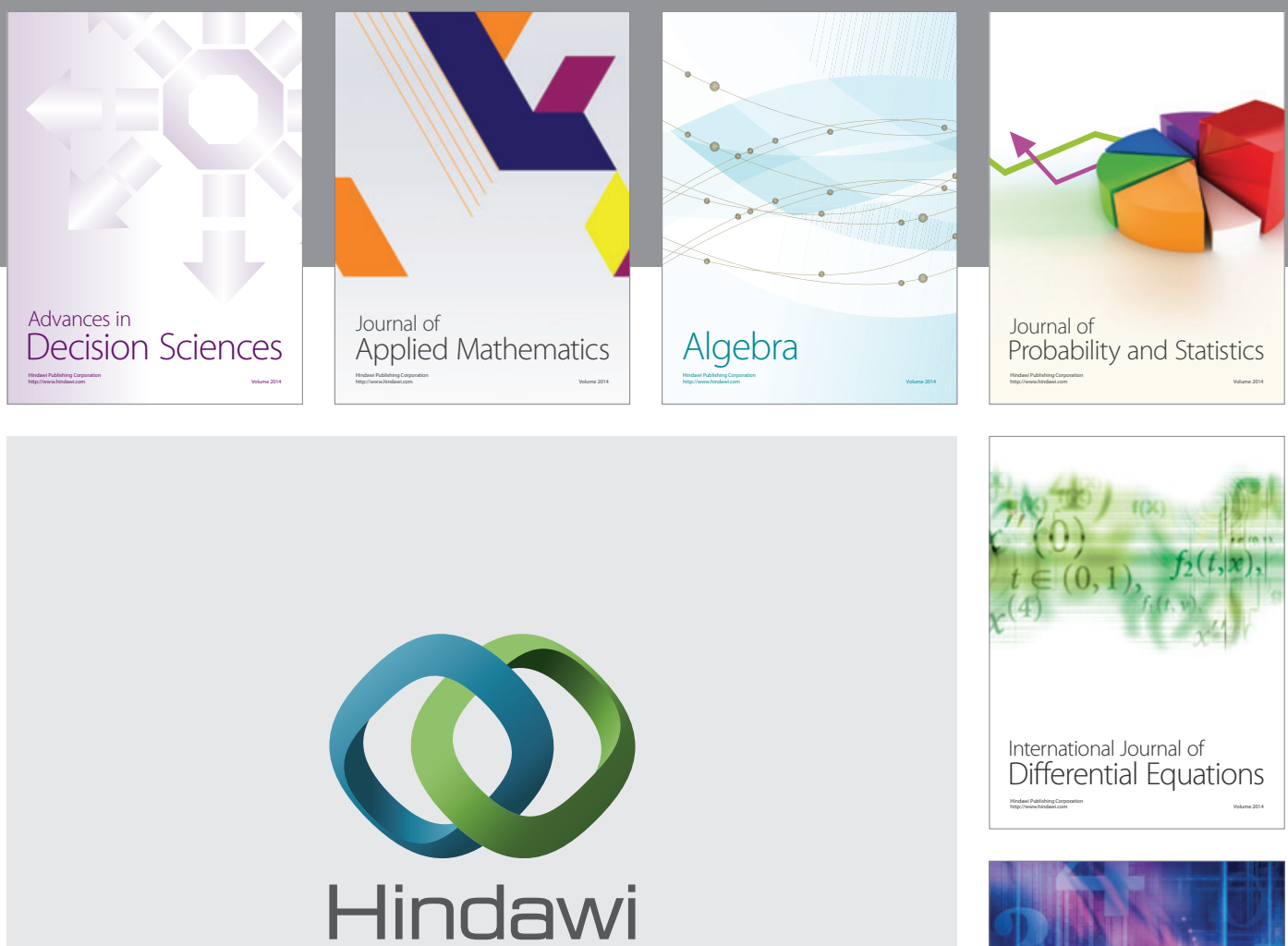

Submit your manuscripts at http://www.hindawi.com
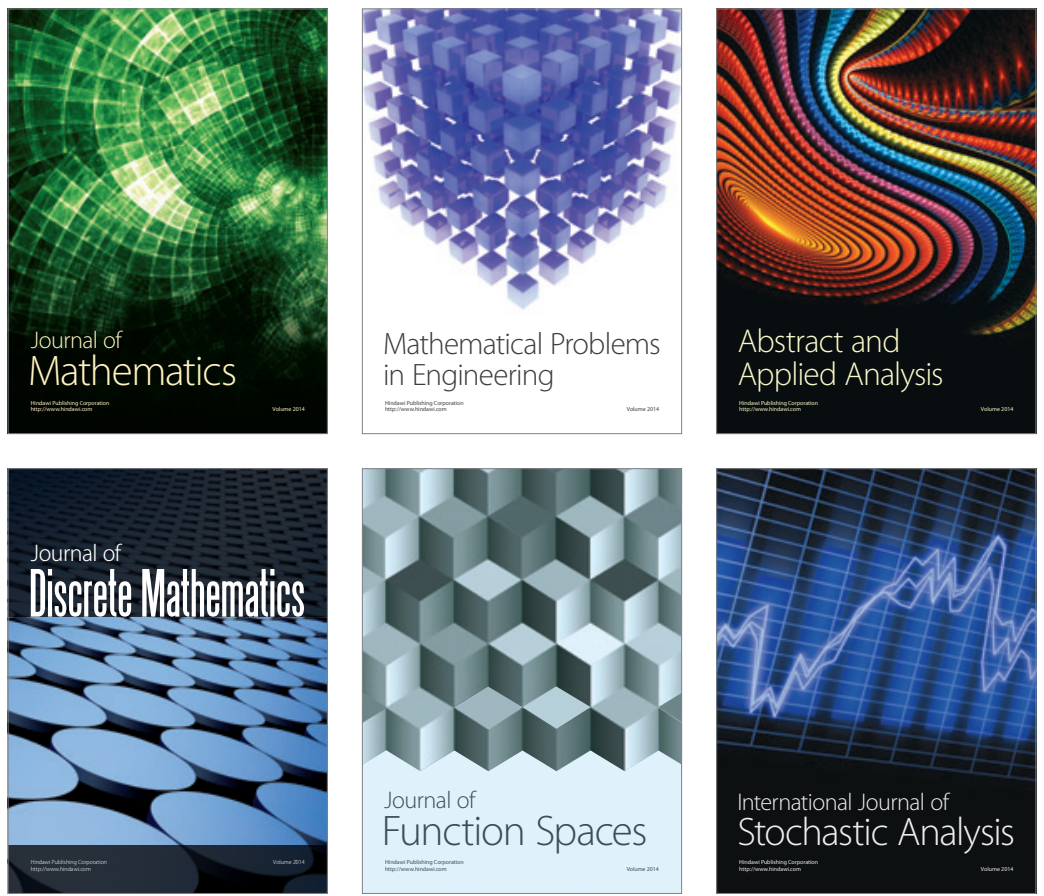

Journal of

Function Spaces

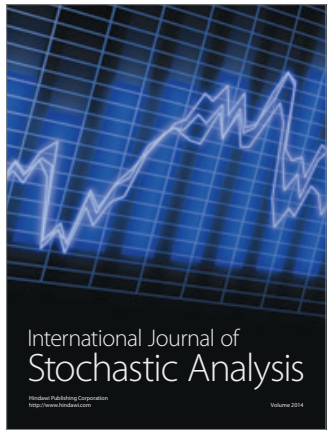

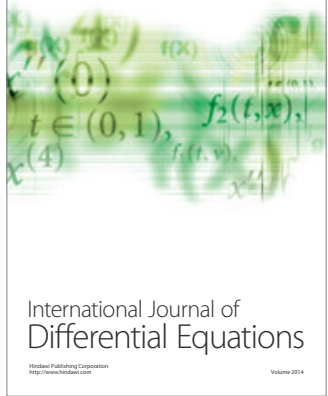
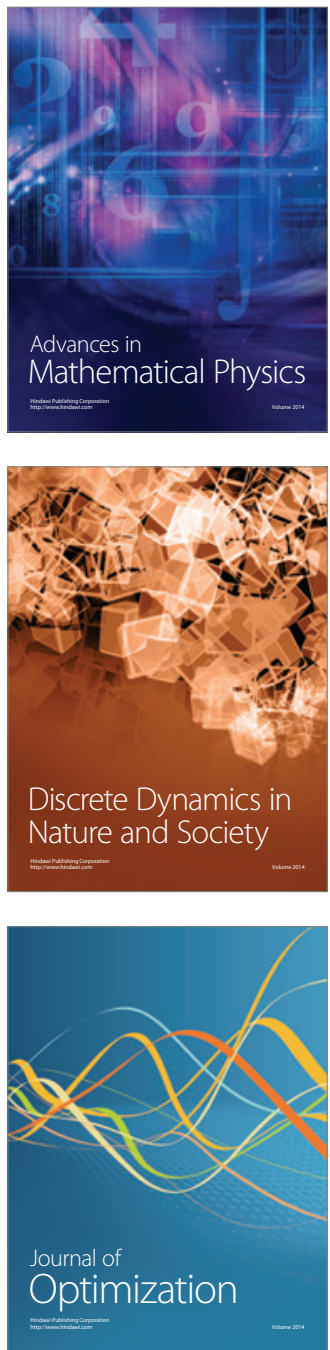